\title{
MediaMTool: Multimedia Content Management Tool
}

\author{
Arghir-Nicolae Moldovan, Catalin Alexandru Moraru and Cristina Hava Muntean
}

\begin{abstract}
With the proliferation of mobile devices, multimedia streaming over wireless networks has increased in popularity. To overcome a number of challenges as well as to enhance mobile users' experience, much research effort has been placed into multimedia content adaptation and personalisation. Supporting adaptive multimedia can pose itself a number of challenges, especially when considering the fast growing rate at which multimedia content is being produced. This paper explores the idea of automatic multimedia content management and authoring to support adaptive multimedia delivery to mobile devices. A multimedia content management tool (MediaMTool) is presented which automatically creates multiple versions of the multimedia clips based on a set of specified multimedia clip features. For testing purposes, MediaMTool was used in conjunction with EcoLearn, a m-learning system that adapts the quality of the educational multimedia clips in order to save battery power on the learner mobile device.
\end{abstract}

Index Terms-multimedia content management, multimedia adaptation, mobile devices, battery power save.

\section{INTRODUCTION}

$\mathrm{F}$ IFTY years ago no one could have envisioned that computers will become indispensable in all the fields of the society. From business to science and from engineering to entertainment it has become more and more obvious that computers play a key role in maintaining and developing interhuman relations.

One of the most significant changes of the last ten years is the huge technological advances and the proliferation of mobile devices such as laptops, PDAs, smartphones, and tablets that are used to access, transfer, manage and produce information. Moreover, there is a clear trend that sees multimedia content production and consumption growing exponentially. Nowadays multimedia content not only that can be displayed by a multitude of mobile devices, but many devices have recording capabilities as well. A large number of applications and services that deliver multimedia content to mobile devices such as mobile IPTV, Video on Demand and video sharing were also developed.

Manuscript received May 6, 2011. This work was supported by IRCSET Embark Postgraduate Scholarship Scheme and ODCSSS (Online Dublin Computer Science Summer School) programme.

A.-N. Moldovan is with the School of Computing, National College of Ireland, Mayor Street, Dublin 1, Ireland(e-mail: amoldovan@student.ncirl.ie).

C.-A. Moraru is with the Faculty of Computing, "Politehnica" University of Timisoara, Vasile Parvan Boulevard, Timisoara ,Romania (e-mail: c.a.moraru@gmail.com).

C. H. Muntean is with the School of Computing, National College of Ireland,Mayor Street, Dublin 1, Ireland (e-mail: cmuntean@ncirl.ie).
However, the mobility characteristics of these devices raise a number of problems associated with the wireless connectivity (e.g. lower bandwidth, increase error rate, etc.), and the multitude of mobile device characteristics (e.g. screen resolution, CPU speed, multimedia formats supported, battery life, etc.). To overcome such problems, and provide users with content that better suits their network conditions and device characteristics, multimedia content personalisation and adaptation solutions are increasingly adopted.

On another side multimedia clips personalisation and adaptation often requires creating multiple versions of the same clip, while knowledge about the network conditions and device characteristics is required in order to create these versions. In this context, automatic multimedia management and authoring solutions are necessary in order to deal with the fast growing rate at which multimedia content is being produced, and to help the content authors preparing the multimedia content for personalisation and adaptation.

This paper presents MediaMTool, a multimedia content management and authoring tool that is suitable for multimedia systems that personalise and adapt the multimedia clips to the user device specific requirements (e.g. device screen resolution, battery power, network bandwidth, etc.). MediaMTool automates multimedia clips management and preparation for personalisation and adaptation, alleviating the content authors of these difficult tasks. To test the capabilities of the proposed solution, MediaMTool was used in conjunction with EcoLearn - an adaptive mobile learning (mlearning) system. EcoLearn delivers adaptive educational multimedia clips, by decreasing the bitrate, with the goal to save the battery power on the learner mobile device, thus assisting the learners to study for a longer period of time.

The remaining of the paper is structured as follows. Section II presents the main approaches for multimedia content management and authoring, as well as for battery power saving in mobile devices. Section III presents the architecture and describes the functionality of the MediaMTool. Section 4 exemplifies how MediaMTool was used in conjunction with EcoLearn and briefly summarises the experimental and subjective tests that were conducted, while Section V concludes the paper.

\section{RELATED WORK}

\section{A. Multimedia Content Management and Authoring}

Content management and content authoring are two concepts that partially overlap, and between which it is difficult to draw a clear distinction line. Broadly speaking 
content management refers to the process of collecting, managing and publishing the content, while content management tools facilitate the tasks by providing automatic features [1]. Content authoring as opposed, is mostly related with the content design, creation and presentation, while authoring tools aim to facilitate these tasks for content authors that lack advanced skills such as programming skills [2].

Different type of digital content can be managed and authored, such as documents, images, multimedia clips, etc. As part of adaptive systems such as Adaptive e-Learning Systems (AeLS), authoring tools are also used to facilitate the creation of the adaptation strategies.

The main approaches for content authoring for multimodal devices can be classified in: single authoring, multiple authoring and flexible authoring [3].

Single authoring involves creating a single version of the content that is valid for all the devices. Various techniques can be used for such purpose, depending on the content type. Device independent multimedia presentations consisting of various bits of information such as text, images, audio and/or video can be created using device independent languages such as, among others, XML, HTML, SMIL, Java or Flash [4]. Single authoring of multimedia clips can be performed using scalable codecs such as Scalable Video Codec (SVC) extension of the H.264/AVC video codec standard [5]. Scalable codecs are designed to overcome the need for creating multiple versions of the same multimedia clip for different mobile devices. Instead the multimedia clip is encoded in a single file containing a base layer and several enhancement layers, from which multiple streams can be generated based on the device characteristics by adding enhancement layers to the base layer. An authoring tool that uses the SMART scalable video coding mechanism is presented in [6].

Multiple authoring involves creating multiple versions of the content targeting different devices or categories of devices, with the obvious drawback of high production and maintenance efforts. For the particular case of multimedia clips, the techniques that can be used for creating the multiple versions range from transcoding to selection and replacement [7]. Transcoding involves converting the multimedia clip to a different format or changing parameters such as for example, the video resolution in order to meet the constraints of different devices. To assist the transcoding, metadata can be defined using existing standards such as MPEG-7 that enables, among others, the content description (e.g. title, bitrate, motion, colours, etc.), and MPEG-21 that enables usage environment description (e.g. device capabilities, network characteristics, user characteristics, etc.) [8]. As opposed to transcoding, multimedia selection and reduction involve changing the content presentation, thus requiring more elaborate pre-processing. Examples of selection techniques include extracting elements from the clips users may be more interested in (e.g. scenes, region of interest (ROI) in frames [9], etc.), while replacement techniques involve replacing elements in a clip with other elements that may be of a different media type (e.g. replacing consecutive scenes in a clip with representative images).

Flexible authoring combines single authoring with multiple authoring. It has the most potential but it also implies the most effort. An example of authoring tool that uses flexible authoring is MEAT [10], which uses multiple authoring for content and single authoring for the test items. MEAT generates SCORM conformable adaptable learning resources and provides authoring for multimodal devices. It has support for multiple media types including PowerPoint presentations, Web Pages, Flash and multimedia clips.

A significant number of visual-based multimedia authoring tools such as Authorware, Director, iShell and MediaWorks are presented in [11]. While such tools enable users with little or no programming experience to create and edit multimedia content, the process is mostly manual and therefore they are not suitable for preparing a very large amount of multimedia content for personalisation and adaptation.

\section{B. Battery Power Saving}

Battery capacity still represents a limited resource of the mobile devices. Also resource-hungry tasks such as playing a multimedia clip streamed over the wireless network can drain very quickly the power from the battery.

Due to these limitations intense research has been done towards extending the battery life of the mobile devices. Various power saving techniques were proposed for the particular case of multimedia streaming to mobile devices over Wireless LANs. Depending on where the battery power is mainly saved, these techniques can be classified in: reception-related techniques that aim to reduce the power consumption of the wireless network card [12], decodingrelated techniques that aim to reduce the power consumption of the CPU [13] and memory [14], and playing-related techniques that mainly focus on the device display [15].

Adaptive multimedia encoding techniques that convert the video to versions that are less power-expensive to be received, decoded and displayed can also be used for reducing the mobile device power consumption. Such a technique is to reencode the clip with a compression technique that requires less bitrate for the same quality or is less computational expensive [16]. Encoding parameters such as resolution, frame rate or bitrate can also be reduced for saving mobile device battery power [17].

The re-encoding can be done on-the-fly by transcoding the video in real time on the server or at an intermediate proxynode before delivering it to the user device [18]. Alternatively, multiple versions can be created off-line for each multimedia clip, in which case the most suitable for a particular user device needs to be selected.

Due to the high number of versions that may need to be created depending on the adaptation goals, automatic multimedia content management and authoring tools are necessary for efficient management, metadata extraction and annotation of these versions so that they can be effectively used in the adaptation process. 


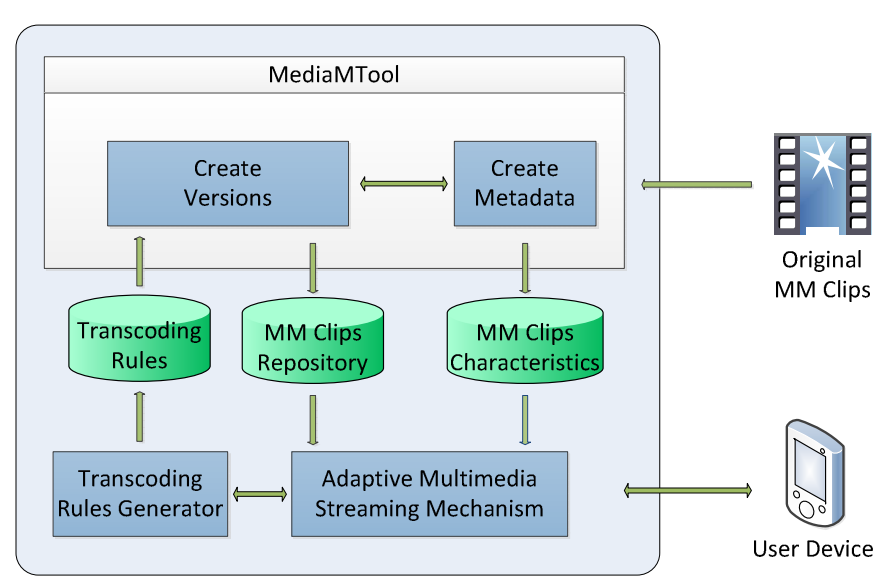

Fig. 1. Block level architecture of MediaMTool

\section{MEDIAMTOOL}

This section describes the proposed multimedia content management and authoring tool - MediaMTool. Content management involves three main phases: creation, preparation and delivery. MediaMTool allows the creation and management of different versions of multimedia clips in order to support personalised and adaptive multimedia delivery. Figure 1 presents the architecture of the MediaMTool at block level and how it communicates with the other components of a multimedia system. When a multimedia clip is uploaded on the server, metadata such as video and audio compression, resolution, frame rate and bitrate is automatically extracted by MediaMTool and saved to a database called generically MM Clips Characteristics. Based on this metadata and on a set of transcoding rules, multiple versions of the multimedia clip are created and metadata is extracted and stored for each of these versions. The transcoding rules are generated automatically by a Transcoding Rules Generator (TRG) based on the specific requirements of the Adaptive Multimedia Streaming Mechanism (AMSM). These versions may have different characteristics depending on the type of adaptation being performed. The adaptation process may be driven by various factors such as the network bandwidth, the device screen resolution, CPU speed, battery power, etc.

A prototype MediaMTool was developed using freely available technologies. The Web Interface was developed using HTML and PHP languages, and was deployed on an Apache Web Server. The interface (see Figure 2) provides functionalities such as uploading multimedia clips to MM Clip Repository, viewing the versions that are created and their metadata, deleting the clips/versions that are not needed anymore. Once a clip is uploaded, metadata is automatically extracted using the Media Info CLI [19] and is stored in a MySQL database (see Figure 3). The metadata consist of the following information regarding the multimedia clip: file name, file format, duration, file size, title, comment, resolution, aspect ratio, video codec, video bitrate, frame rate, audio codec and audio bitrate. After metadata is extracted from the original clip the tool continues with the transcoding

\begin{tabular}{|c|c|c|}
\hline Location $\quad:$ http://192.168.1.16/MM_T $\mathrm{s}$ & START EDIT & SAVE \\
\hline \multicolumn{3}{|l|}{ Videos I Videos Versions I Transcodina Settinas } \\
\hline Upload a new video: Choose File No file chosen & & Upload \\
\hline \multicolumn{3}{|l|}{ Uploaded videos } \\
\hline \multicolumn{3}{|l|}{ Delete } \\
\hline$\square$ File Name & Size $[M B]$ & Path \\
\hline 1-01 Adiabate.mp4 & 13 & ../Videos/1-01 Adiabate.mp4 \\
\hline 1-01 Corals in Crisis $1 . \mathrm{mp} 4$ & 29 & ../Videos/1-01 Corals in Crisis 1.1 \\
\hline $1-01 \mathrm{M} 82$ in 60 Seconds.m4v & 26 & .../Videos/1-01 M82 in 60 Seconds \\
\hline 1-02 Herramientas de seleccion (I).mp4 & 7 & ../Videos/1-02 Herramientas de s \\
\hline$\square$ 1-06 Transcription_writing the mess.m $4 \mathrm{v}$ & Iv 27 & .../Videos/1-06 Transcription_wri \\
\hline$\square$ 1-107 LP23_Const Parameters - ipod.m4v & $4 v 7$ & .../Videos/1-107 LP23_Const Para \\
\hline$\square$ ACUmobile 1-iPhone.m4v & 6 & .../Videos/ACUmobile 1-iphone.m. \\
\hline$\square$ QUEST Quiz_Sewage.m4v & 17 & .../Videos/QUEST Quiz_Sewage.m \\
\hline
\end{tabular}

Fig. 2. Web interface of MediaMTool

\begin{tabular}{|c|c|}
\hline Location & http://192.168.1.16/MM_T START EDIT SAVE \\
\hline \multicolumn{2}{|l|}{ Back to Videos } \\
\hline \multicolumn{2}{|l|}{ Metadata } \\
\hline \multirow{4}{*}{$\begin{array}{l}\text { File Name } \\
\text { Format } \\
\text { Duration [s] } \\
\text { File Size [MB] }\end{array}$} & ../Videos/Wildlife.wmv \\
\hline & Windows Media \\
\hline & 30 \\
\hline & 25 \\
\hline \multirow{2}{*}{$\begin{array}{l}\text { Title } \\
\text { Comment }\end{array}$} & Wildlife in HD \\
\hline & Footage: Small World Productions, Inc; Tourism New Zealand I Producer: \\
\hline Height & 1280 \\
\hline \multirow{2}{*}{$\begin{array}{l}\text { Width } \\
\text { Aspect Ratio }\end{array}$} & 720 \\
\hline & $16: 9$ \\
\hline \multirow{2}{*}{$\begin{array}{l}\text { Video Codec } \\
\text { Bitrate [kbps] }\end{array}$} & VC-1 \\
\hline & 5942 \\
\hline \multirow{3}{*}{$\begin{array}{l}\text { Framerate } \\
\text { Audio Format } \\
\text { Audio Codec }\end{array}$} & 30 \\
\hline & WMA \\
\hline & 161 \\
\hline \multicolumn{2}{|c|}{ Audio Bitrate [kbps] 192} \\
\hline
\end{tabular}

Fig. 3. Metadata automatically extracted for a multimedia clip

process, for creating multiple versions of the multimedia clip according to specific transcoding rules. FFmpeg CLI [20] was used for transcoding since it can convert, stream and play almost any video format.

The automatic management and preparation for adaptation of the multimedia content, that alleviates the content authors of these tasks, are main benefits brought by the MediaMTool tool. MediaMTool is independent from any specific domain meaning that it can be used to manage multimedia content both in client-server applications such as adaptive m-learning systems and desktop specific applications. This is because it was designed as a generic multimedia management framework. The proposed tool is independent from the adaptation algorithm. Therefore, when new criteria of 
adaptation are introduced little to no modifications will be necessary in MediaMTool, for creating the new versions. Although the prototype that was implemented was exemplified only on multimedia clips, the solution can be easily extended to include other types of media content such as images and audio clips.

\section{Mediamtool Used as Multimedia Content AUTHORING AND MANAGEMENT FOR THE ECOLEARN SYSTEM}

\section{A. EcoLearn Overview}

EcoLearn [21] is an adaptive m-learning system that adapts the educational multimedia clips, with the goal to reduce the battery power consumption on the learner mobile device, assisting the learner to study for a longer period of time. Due to the high diversity of mobile devices learners may use, EcoLearn groups the users' devices into several classes based on their screen resolution. A video profile consisting of a reference resolution and a reference bitrate is associated to each device class. The reference values are selected to offer excellent user perceived quality level even for multimedia clips with high level of motion content, when streamed to the corresponding mobile devices.

For each educational multimedia clip, multiple versions with different reference bitrate and reference resolution (corresponding to a device class) are generated using the MediaMTool. The reference-based versions $M M_{X}^{i}$ corresponding to a multimedia clip $M M^{i}$ are represented in an abstract form as follows:

$M M_{X}^{i} \in\left\{\left(\operatorname{RefRES}_{X}, \operatorname{RefBR_{X}}\right)\right\}, X=\overline{1, N}$

where $X$ indexes $N$ particular reference-based versions corresponding to $N$ classes of mobile devices.

Each reference version has a unique video resolution $\operatorname{RefRES}_{X}$ and a unique video bitrate $\operatorname{RefBR} R_{X}$. The two parameters vary across video references but not across multimedia clips. For example in (2), $M M_{1}^{1}$ and $M M_{1}^{2}$ are reference versions that were created from two different multimedia clips, $M M^{1}$ and $M M^{1}$ respectively. The two versions correspond to the same class of mobile devices, thus they have the same resolution and bitrate (e.g. 640x360 pixels and $1200 \mathrm{Kbps}$ respectively). As opposed, $M M_{1}^{1}$ and $M M_{2}^{1}$ are reference versions created from the same multimedia clip $M M^{1}$, but since they correspond to different device classes they have different resolution and bitrate.

$$
\begin{aligned}
& M M_{1}^{1}=(640 \times 360 \text { pixels, } 1200 \mathrm{Kbps}) \\
& M M_{1}^{2}=(640 \times 360 \text { pixels, } 1200 \mathrm{Kbps}) \\
& M M_{2}^{1}=(856 \times 480 \text { pixels, } 2000 \mathrm{Kbps})
\end{aligned}
$$

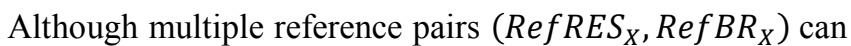
be defined in order to cover a broad spectrum of devices, in practice it is not always possible to create reference-based versions corresponding to all of these pairs from each multimedia clip. The number of reference-based versions $N$
TABLE I

MAPPING OF OBJECTIVE VIDEO QUALITY TO SuBJECTIVE QUALITY

\begin{tabular}{lll}
\hline \hline \multicolumn{1}{c}{ MOS } & \multicolumn{1}{c}{ PSNR [dB] } & \multicolumn{1}{c}{ SSIM } \\
\hline 5 (Excellent) & $\geq 45$ & $\geq 0.99$ \\
4 (Good) & $\geq 33 \&<45$ & $\geq 0.95 \&<0.99$ \\
3 (Fair) & $\geq 27.4 \&<33$ & $\geq 0.88 \&<0.95$ \\
2 (Poor) & $\geq 18.7 \&<27.4$ & $\geq 0.5 \&<0.88$ \\
1 (Bad) & $<18.7$ & $<0.5$ \\
\hline \hline
\end{tabular}

that can be created from a particular multimedia clip $M M^{i}$ depends on the original quality of the clip, $\operatorname{RefRES_{X}}$ and $R e f B R_{X}$ being limited to the original video resolution and video bitrate of that clip.

To save mobile device battery power, EcoLearn decreases the video bitrate to lower levels than the excellent quality supported through the reference bitrate. To create lower bitrate versions for each reference resolution, MediaMTool works in conjunction with BitDetect [22], an automatic mechanism that detects a lower bitrate threshold $\left(\operatorname{MinBR}_{X}^{i}\right)$, that still offers a good level of user perceived quality as compared to the excellent quality level offered by the reference bitrate $R e f B R_{X}$. For a particular reference resolution $\operatorname{RefRES} S_{X}$ the $M i n B R_{X}^{i}$ bitrate threshold may vary across different multimedia clips depending on their characteristics such as the level of motion content, colours used, etc. Therefore, the $\operatorname{Min} B R_{X}^{i}$ threshold is detected individually for each referencebased version $M M_{X}^{i}$ of each multimedia clip $M M^{i}$.

BitDetect uses two objective metrics, Signal-to-Noise Ratio (PSNR) and Structural Similarity Index (SSIM) for video quality assessment. For each reference-based version $M M_{X}^{i}$ of a multimedia clip, new versions are automatically created by MediaMTool by gradually decreasing the bitrate from the reference bitrate $\operatorname{RefRES_{X}}$ with a fixed step $S t e p_{X}$ associated to the reference pair $\left(\operatorname{RefRES} S_{X}, \operatorname{RefB} R_{X}\right)$. The quality of each version is estimated by BitDetect by measuring the PSNR and SSIM metrics and mapping the results on a 1 to 5 subjective scale proposed in [23] (see Table I). Additional details on how the bitrate threshold is detected by BitDetect can be found in [22].

The multitude of versions $M M_{X j}^{i}$ with a particular reference resolution $\operatorname{RefRES} S_{X}$, created from a multimedia clip $M M^{i}$ is represented in (3).

$M M_{X j}^{i} \in\left\{\left(\right.\right.$ RefRES $_{X}$, Bitrate $\left.\left._{X j}^{i}\right)\right\}, j=\overline{1, P}$

where

$$
\begin{gathered}
\text { Bitrate }_{X j}^{i} \in\left\{\operatorname{RefBR}_{X}, \operatorname{RefBR}_{X}-\operatorname{Step}_{X}, \operatorname{RefBR_{X}-}\right. \\
\left.-2 \cdot \operatorname{Step}_{X}, \ldots, \operatorname{MinBR}_{X}^{i}\right\}
\end{gathered}
$$

and $j$ indexes $P$ versions with a $\operatorname{RefRES} S_{X}$ resolution created from the multimedia clip $M M^{i}$.

In practice it is not feasible and useful to store all the versions created for each reference resolution corresponding to a device class. Therefore, for each reference resolution RefRES $S_{X}$ corresponding to a device class, EcoLearn stores only two versions that were created from the multimedia clip $M M^{i}: M M_{X}^{i}$ that has a $\operatorname{RefBR} R_{X}$ bitrate, and $M M_{X P}^{i}$ that has 


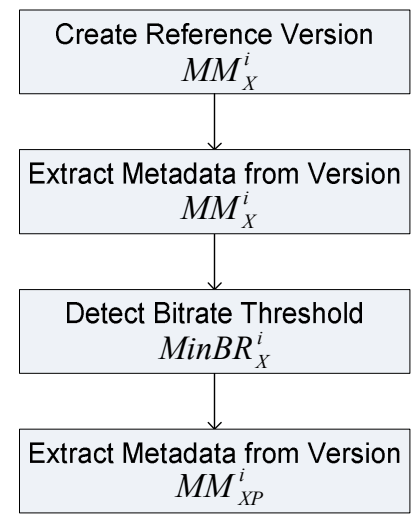

Fig. 4. Creating the two versions corresponding to a device class for a multimedia clip $M M^{i}$.

a MinBR $R_{X}^{i}$ bitrate, as detected by BitDetect. The process deployed by EcoLearn in order to create the two versions for a reference resolution $\operatorname{RefRES}_{X}$ is presented in Figure 4. The four steps are repeated for as many device classes as desired and possible. Additionally, metadata is automatically extracted and stored for the two versions corresponding to each device class, using the MediaMTool.

When a user with a particular mobile device requests a multimedia clip, EcoLearn selects the version having the most suitable resolution for that device's screen resolution. If the battery level is detected as being too low for the entire clip to be fully displayed, the adaptive mechanism of the EcoLearn system switches to the lower quality version having the same resolution but the lower bitrate, as detected by BitDetect.

\section{B. Tests and Results}

The MediaMTool was tested in order to assess its correct functionality when used in conjunction with the EcoLearn adaptive e-learning system. The versions correctly created by MediaMTool were used during experimental and subjective tests that were conducted in order to assess the benefits provided by EcoLearn adaptive e-learning system in terms of battery power saving and user perceived quality. The tests were conducted in an e-learning/m-learning context, but the tool and the adaptive mechanism can be easily applied in the general context of multimedia streaming to mobile devices.

Eight educational multimedia sequences up to 30 seconds long were used for this testing. The sequences were selected so to cover a broad spectrum of educational clips in terms of content type and dynamicity. These sequences correspond to the following categories of educational multimedia clips: screencasts, slideshows, computer generated animations, 3D game and virtual world recordings, interviews, lecture recordings, lab demos and documentaries. Additional details about the eight test sequences can be found in [21]. For each sequence two versions having the same video resolution 640x360 were automatically created using the MediaMTool. H.264/MPEG-4 AVC video codec was used. For all test sequences the bitrate of the first version (the reference one), was selected as $1200 \mathrm{Kbps}$ following recommendations found in [24], [25] and [26] for encoding multimedia content, assuming that the value offers an excellent quality level even for the clips with the highest level of dynamicity. Using the BitDetect mechanism the bitrate of the second version was detected individually for each sequence as follows: $512 \mathrm{Kbps}$ for the sequence corresponding to documentaries, and 256 Kbps for the other seven test sequences.

To assess the battery saving capabilities, the 16 clips corresponding to the 8 test sequences were streamed wirelessly to an HP iPAQ 214 PDA, and the device power consumption was measured for each case. The results for a $20 \%$ battery discharge are presented in Figure 5. The results show that depending on the clip characteristics, between $13 \%$ and up to almost $17 \%$ increase in battery life can be achieved by reducing the bitrate from the reference bitrate to the threshold recommended by BitDetect. Additional details on the experimental results can be found in [21].

For the subjective assessment, the Absolute Category Rating [27] method for video quality assessment of multimedia applications was used. The 21 subjects participating in the subjective assessment were asked to rate their perceived quality of the eight low bitrate versions on a five-level quality scale, where 1 is "Bad" and 5 is "Excellent". By averaging the ratings across the 21 subjects, the Mean Opinion Scores (MOS) for the eight test sequences were obtained. The average MOS across the eight test sequences was 4.1 , while individually only two sequences were rated with less than 4 (3.6 and respectively 3.9) More details about subjective assessment can be found [22].

\section{CONCLUSIONS}

This paper introduced MediaMTool, a content management tool that is suitable for systems that perform adaptive multimedia streaming. This tool may be used to create multiple versions of the multimedia clips required by an adaptive multimedia streaming mechanism. The paper has exemplified how MediaMtool was used in conjunction with EcoLearn, an adaptive e-learning system that delivers adaptive educational multimedia clips for device battery consumption optimisation. EcoLearn incorporates BitDetect, a mechanism for detecting good quality bitrate thresholds for enabling bitrate-based adaptation of multimedia clips.

Experimental and subjective tests conducted using a number of educational multimedia sequences, have shown that by using the multimedia versions correctly created by the tool, significant device battery power can be saved while at the same time maintaining a good level of user perceived quality. Up to $17 \%$ in streaming time has been achieved by reducing the bitrate from the reference bitrate to the one recommended by the BitDetect mechanism. The user-time was gained with no significant alteration of the viewing experience. Subjective assessments have shown that the overall average MOS score was 4.1 corresponding to Good on a 1 to 5 subjective scale. 


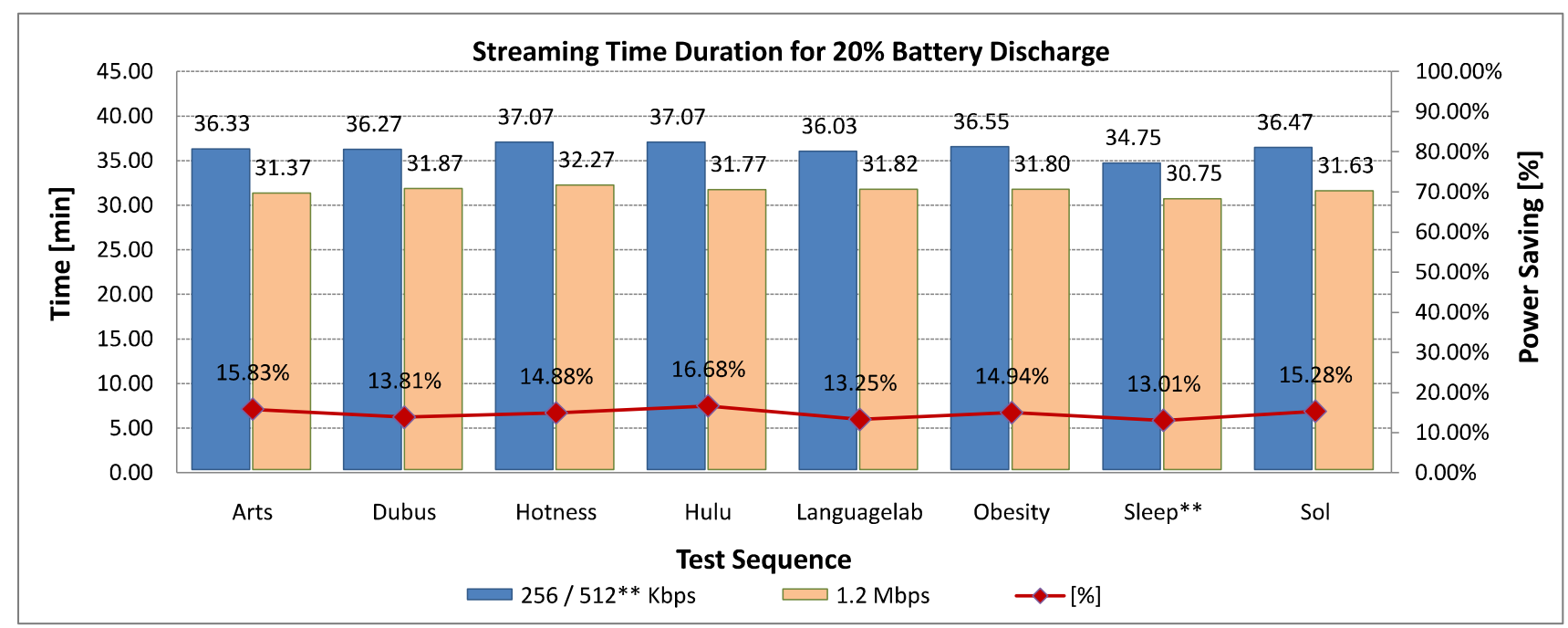

Fig. 5. Streaming time duration for the eight video sequences during a $20 \%$ battery discharge

\section{REFERENCES}

[1] G. Pullman and B. Gu, "Guest Editors' Introduction: Rationalizing and Rhetoricizing Content Management," Technical Communication Quarterly, vol. 17, no. 1, pp. 1-9, 2008.

[2] T. Arndt and E. Katz, "Visual Software Tools for Multimedia Authoring," Journal of Visual Languages \& Computing, vol. 21, no. 3, pp. 184-191, 2010.

[3] R. Simon, F. Wegscheider, and K. Tolar, "Tool-supported Single Authoring for Device Independence and Multimodality," in Proceedings of the 7th International Conference on Human Computer Interaction with Mobile Devices \& Services (MobileHCI '05), Salzburg, Austria, pp. 91-98, 2005.

[4] R. Mugwanya and G. Marsden, "Mobile Learning Content Authoring Tools (MLCATs): A Systematic Review," in E-Infrastructures and EServices on Developing Countries, vol. 38, A. Villafiorita, R. Saint-Paul, and A. Zorer, Eds. Springer Berlin Heidelberg, pp. 20-31, 2010.

[5] H. Schwarz, D. Marpe, and T. Wiegand, "Overview of the Scalable Video Coding Extension of the H.264/AVC Standard," IEEE Transactions on Circuits and Systems for Video Technology, vol. 17, no. 9, pp. 1103-1120, 2007.

[6] X.-S. Hua and S. Li, "Personal media sharing and authoring on the web," in Proceedings of the 13th Annual ACM International Conference on Multimedia, New York, NY, USA, pp. 375-378, 2005.

[7] S.-F. Chang and A. Vetro, "Video Adaptation: Concepts, Technologies, and Open Issues," Proceedings of the IEEE, vol. 93, no. 1, pp. 148-158, 2005

[8] H. Ali, "Transcoding for Content Management Systems". Technology Gjøvik University College, 2009. [Online]. Available: http://www.ansatt.hig.no/kjellr/imt4951/projects/20091202_imt4951_ali/ report.pdf

[9] B. L. Velammal and P. A. Kumar, "Dynamic Content Adaptation for Multimedia Content in Handheld Devices," Journal of Computer Science and Engineering, vol. 2, no. 1, 2010. [Online]. Available: http://sites.google.com/site/jcseuk/volumes/V2-I1-P1-5.pdf

[10] Y. H. Kuo and Y. M. Huang, "MEAT: An Authoring Tool for Generating Adaptable Learning Resources," Journal of Educational Technology \& Society, vol. 12, no. 2, pp. 51-68, 2009.

[11] T. H. Kaskalis, T. D. Tzidamis, and K. Margaritis, "Multimedia Authoring Tools: The Quest for an Educational Package," Educational Technology \& Society, vol. 10, no. 3, pp. 135-162, 2007.

[12] Y. He and R. Yuan, "A Novel Scheduled Power Saving Mechanism for 802.11 Wireless LANs," IEEE Transactions on Mobile Computing, vol. 8, no. 10, pp. 1368-1383, 2009.

[13] Z. Cao, B. Foo, L. He, and M. van der Schaar, "Optimality and Improvement of Dynamic Voltage Scaling Algorithms for Multimedia Applications," IEEE Transactions on Circuits and Systems Part I: Regular Papers, vol. 57, no. 3, pp. 681-690, 2010.
[14] K. Amiri, A. Khajeh, A. M. Eltawil, and F. J. Kurdahi, "Process variation aware transcoding for low power H.264 decoding," in Proceedings of 8th IEEE Workshop on Embedded Systems for Real-Time Multimedia (ESTIMedia 2010), Scottsdale, AZ, USA, pp. 90-96, 2010.

[15] M. Dong, Y.-S. K. Choi, and L. Zhong, "Power Modeling of Graphical User Interfaces on OLED Displays," in Proceedings of the 46th Annual Design Automation Conference, New York, NY, USA, pp. 652-657, 2009.

[16] C.-H. Lin, J.-C. Liu, and C.-W. Liao, "Energy analysis of multimedia video decoding on mobile handheld devices," Computer Standards \& Interfaces, vol. 32, no. 1-2, pp. 10-17, 2010.

[17] A.-N. Moldovan and C. H. Muntean, "Personalisation of the multimedia content delivered to mobile device users," in Proceedings of IEEE International Symposium on Broadband Multimedia Systems and Broadcasting (BMSB '09), Bilbao, Spain, 2009.

[18] S. Mohapatra, N. Dutt, A. Nicolau, and N. Venkatasubramanian, "DYNAMO: A Cross-Layer Framework for End-to-End QoS and Energy Optimization in Mobile Handheld Devices," IEEE Journal on Selected Areas in Communications, vol. 25, no. 4, pp. 722-737, 2007.

[19] MediaInfo, "MediaInfo supplies technical and tag information about a video or audio file." [Online]. Available: http://mediainfo.sourceforge.net/en.

[20] FFmpeg Team, "FFmpeg - a complete, cross-platform solution to record, convert and stream audio and video." [Online]. Available: http://www.ffmpeg.org/.

[21] A.-N. Moldovan, A. Molnar, and C. H. Muntean, "EcoLearn: Battery Power Friendly e-Learning Environment for Mobile Device Users," in Learning-Oriented Technologies, Devices and Networks - Innovative Case Studies, A. Lazakidou and I. El Emary, Eds. Saarbrücken, Germany: LAP LAMBERT Academic Publishing, pp. 273-296, 2011.

[22] A.-N. Moldovan and C. H. Muntean, "Subjective Assessment of BitDetect - A Mechanism for Energy-Aware Adaptive Multimedia," Submitted to IEEE Transactions on Broadcasting, 2011.

[23] T. Zinner, O. Abboud, O. Hohlfeld, T. Hossfeld, and P. Tran-Gia, "Towards QoE Management for Scalable Video Streaming," in Proceedings of the 21th ITC Specialist Seminar on Multimedia Applications - Traffic, Performance and QoE, Miyazaki, Japan, 2010.

[24] M. O'Connell and J. Smith, A guide to working with m-learning standards. Australian Flexible Learning Framework, 2007.

[25] A. Kapoor, "Dynamic streaming on demand with Flash Media Server 3.5 Adobe Developer Connection," 12-Jan-2009. [Online]. Available: http://www.adobe.com/devnet/flashmediaserver/articles/dynstream_on_ demand.html.

[26] Apple Inc., "Apple - QuickTime - Tutorials - Creating Amazing Video Content with H.264." [Online]. Available: http://www.apple.com/mx/quicktime/tutorials/h264.html.

[27] ITU-T, "P.910: Subjective video quality assessment methods for multimedia applications". Geneva, Switzerland: International Telecommunication Union, 2008. 